\title{
X-Ray "Knife" as a Submicrometer Tool for Studying Focusing Lens Quality
}

\author{
A. N. Artemiev ${ }^{a}$, A. G. Maevskii ${ }^{a}$, N. A. Artemiev ${ }^{b}$, A. A. Demkiv ${ }^{a}$, Yu. I. Dudchik ${ }^{c}$, \\ A. V. Zabelin ${ }^{a}$, B. Ph. Kirillov ${ }^{a}$, V. V. Kvardakov ${ }^{a}$, F. F. Komarov ${ }^{c}$, \\ O. V. Naida ${ }^{a}$, and A. V. Porokhova ${ }^{a}$ \\ ${ }^{a}$ Russian Research Centre Kurchatov Institute, pl. Kurchatova 1, Moscow, 123182 Russia \\ ${ }^{b}$ Laboratoire d'Optique Appliquée, ENSTA, CNRS UMR7639, Ecole Polytechnique, \\ Chemin de la Hunire, 91761 Palaiseau, France \\ ${ }^{c}$ Sevchenko Institute of Applied Physical Problems, Belarussian State University, ul. Kurchatova 7, Minsk, 220064 Belarus
}

Received March 24, 2008

\begin{abstract}
A mobile x-ray "knife" developed by the authors is described. The device is intended for determining the profile of the spatial intensity distribution in the ionizing radiation beam. Its automated motion system combines two actuator types. Significant displacements are performed using a goniometric module with a piezoelectric actuator. The angular step of this module is 1 arc second, which corresponds to a linear displacement of $0.97 \mu \mathrm{m}$. As a "fine" drive, a rotating module with a piezoelectric column is used. Its linear step can be varied from 0.01 to $1 \mu \mathrm{m}$ at a total number of steps of 1000 . The x-ray knife was experimentally tested at the Kurchatov Center of Synchrotron Radiation for diagnostics of the parameters of a short-focus X-ray lens at a photon energy of $18 \mathrm{keV}$. In the vertical direction, the width of the profile for the intensity distribution in the beam formed by the lens was $2.4 \mu \mathrm{m}$.
\end{abstract}

\section{DOI: $10.1134 / \mathrm{S} 1027451008060098$}

\section{INTRODUCTION}

Recently, highly local studies have had an increasingly important place in works with synchrotron radiation. The locality in such studies is provided by various methods of radiation focusing. An additional impetus to such studies was imparted by the advent of x-ray refractive [1] and Fresnel lenses [2]. The majority of relevant publications were performed using the third-generation synchrotron radiation (SR) sources, i.e., giant and very expensive electron rings, the ESRF (France) and Spring-8 (Japan). Nevertheless, studies using focusing elements, i.e., $x$-ray refractive lenses, were also begun at the Kurchatov SR source (Moscow), whose brightness is significantly lower than that of the above sources.

Beams with a transverse size of the order of $100 \mathrm{~nm}$ are already used in the world [3], and measurement of the intensity profile in such beams becomes an independent problem. Certainly, an x-ray film can be used as a recorder [4, 5]. However, the film has a number of obvious disadvantages, such as the strong dependence of results on the film's "age," the development time and temperature. These hard-to-control factors cause one to make a number of extraordinary operations, i.e., fixing several images on a single film, performance of control exposures, and others. Moreover, high-resolution (of the order of a micrometer) x-ray films are not frequently commercially produced. Such photographic materials have a low sensitivity, and their processing takes a long time. Furthermore, precision digitization of halftone photographic images is not quite a trivial problem. Thus, there is a pronounced contradiction between the modern X-ray source (SR source) and the archaic recording method (using X-ray films).

In the world practice, a modern and fast recording device is the so-called x-ray "knife" [6]. This device is intended for determining the profile of the spatial intensity distribution in the ionizing radiation beam. The device's operating principle is as follows. A opaque screen for measured radiation is gradually introduced into the beam under study. The detector records radiation scattered by this screen. The derivative of the measured scattering intensity curve yields a one-dimensional beam profile. The use of the knife removes a number of disadvantages inherent to the x-ray film. During operation of an automated system, the result is obtained rapidly and immediately in the digital form.

Among the disadvantages in comparison with the film is that the knife yields a one-dimensional pattern. If the ellipse's major axes are oriented correctly, i.e., horizontally and vertically, errors in measurements using the knife instead of the film do not arise. If the source ellipse is inclined, the knife measures the ellipse projection onto the vertical direction. On the other hand, namely this projection controls the resolution of $\mathrm{x}$-ray monochromators, since their rotation axis is, as a 


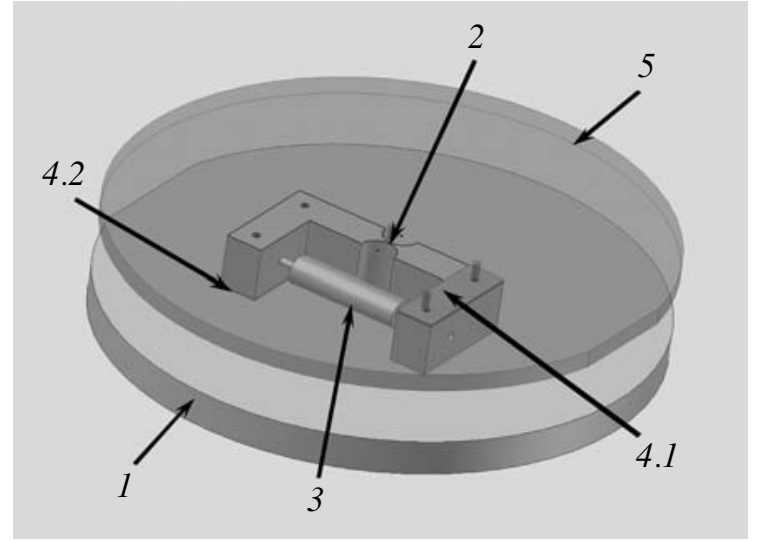

Fig. 1. Schematic diagram of the rotating module with piezoelectric column: (1) module bottom base, (2) bending element, (3) piezoelectric column, (4.1) and (4.2) spacers, and (5) top base.

rule, horizontal. Therefore, information obtained using the knife is exhausting for SR users.

In this paper, we describe a version of the x-ray knife developed, fabricated, and tested at the Kurchatov SR source. As an x-ray optical element forming a diagnosed $\mathrm{x}$-ray beam, a multielement refractive $\mathrm{x}$-ray lens developed at the Sevchenko Institute (Belarus) was used.

\section{DESIGN FEATURES OF THE X-RAY KNIFE}

The problem of fabricating our own knife was rather complex. The requirement of a high spatial resolution varying within $0.01-1 \mathrm{~mm}$ implies the presence of an even and "smooth" x-ray shade. Hence, roughnesses at the knife edge should be smaller than the required spatial resolution by at least an order of magnitude. The decision which seems obvious at first sight, a polished plane face [6] is not unique. Since such a face should be strictly parallel to the SR beam (otherwise, a penumbra will arise due to parasitic passage of $x$-rays through face edges), the use of a cylindrical drum is a simpler design. Cylinder treatment to a mirror finish is very simple. Estimations show that shade spreading due to the passage of x-rays with energy $E_{0}=10 \mathrm{keV}$ in the bronze cylinder $20 \mathrm{~mm}$ in diameter does not exceed $1-1.5 \mu \mathrm{m}$.

The device we developed, i.e., the x-ray knife, allows one to rapidly bring an opaque screen to the beam edge and then to begin to measure the profile by small steps. As a "coarse" drive, we used a previously developed goniometric module with a piezoelectric actuator [7]. The angular step of this module is 1 arc second. For a used 202-mm shoulder, this step corresponds to a linear displacement of $0.97 \mu \mathrm{m}$.

For a "fine" drive, we designed a scanning module with a piezoelectric column. The schematic representation of the scanning module of fine displacement is shown in Fig. 1. The module's top base 5 can rotate at a small angle with respect to the bottom base. Bending element 2 with a thin partition separating its bulk parts is placed between the bottom and top bases. Piezoelectric column 3 is fixed between the bending element shoulders. As the piezoelectric column length is varied, bulk parts of the bending element slightly rotate with respect to each other. The module's top base is fixed on one of the bulk parts of the bending element via spacer 4.1. The module's bottom base is attached to another part of the bending element via spacer 4.2.

The piezoelectric column itself is assembled from several hundred thin piezoelectric disks. The entire range of the piezoelectric column length variation is $50 \mu \mathrm{m}$ at a dc reference voltage of $300 \mathrm{~V}$. The piezoelectric column is controlled by an automated unit. The entire rotation range of the top base with respect to the bottom base is 200 arc seconds. The reference voltage can be decreased to 150 and $75 \mathrm{~V}$. The displacement discreteness is controlled by a digital-to-analog converter; its maximum value is 1024 steps. The piezoelectric column, as well as all other equipment of the experimental testbed, is controlled by a computer with a CAMAC system. An advanced software allowing remote control of all equipment was developed.

Combining the reference voltage of the piezoelectric column and the length of the shoulder on which the knife is installed, various unit linear displacement to micrometer fractions can be performed. A BDS-10 commercial device is used as a detector, which incorporates a photomultiplier and a $\mathrm{NaI}(\mathrm{Tl})$ scintillator. Figure 2 shows the general view of the x-ray knife installed in a protective housing in the REFRA station [8] of the Kurchatov SR Center.

\section{DESIGN FEATURES OF THE X-RAY MICROCAPILLARY REFRACTVE LENS}

The $x$-ray refractive lens developed in the Sevchenko Institute [9-11] is a glass microcapillary with a specified number of spherical epoxy microlenses within it. The radius of an individual microlens is identical to the capillary channel radius. Individual microlenses were formed by sequential blowing of air bubbles in the capillary preliminarily filled with proper liquid material. Therefore, such a lens in some papers is referred to as a "microcapillary" or "bubble" lens $[12,13]$. Figure 3 shows the lens micrograph. The lens consists of 220 spherical microlenses with a curvature radius of $100 \mu \mathrm{m}$ each. The small radius and the large number of microlenses provided a short focal length (of the order of $10 \mathrm{~cm}$ for $10-\mathrm{keV}$ radiation). Such a focal length provides a large ratio of shoulders, hence, a small focal spot. Moreover, the lens is characterized by a high magnification factor, which is important for microobject imaging. The small average atomic number $Z$ of epoxy components provides a low absorbance, hence, a high "gain" of the lens. This circumstance and the smallness of the focal spot make these lenses more efficient in the 
scanning x-ray microscopy method. Surface tension forces causing the spherical shape of the lens provide its surface smoothness providing a high image quality. Glass capillaries providing the lens basis represent a mass product with a high degree of perfection. Therefore, it can be expected that the problem of coaxiality of elementary lenses in the compound lens is solved with high accuracy.

An important factor is that $\mathrm{x}$-ray optical parameters of lenses fabricated by the above technique were repeatedly studied using synchrotron and laboratory radiation sources [10-13]; it was found that such lenses indeed allow the formation of micrometer x-ray beams. Furthermore, theoretical and measured parameters of lenses showed good agreement, which makes it possible to consider them as reference ones.

\section{FEATURES OF MICROCAPILLARY LENS ADJUSTMENT}

The used microcapillary lens has a rather small geometrical aperture; therefore, its alignment along the SR beam presents certain difficulties in contrast to positioning of lenses with large apertures, e.g., Plexiglas lenses [14]. The entrance aperture of the latter was $\sim 1 \mathrm{~mm}$, and the lens was oriented by measuring geometrical parameters of the x-ray beam passed along a triangular groove of the compound lens base.

In the case of a microcapillary lens, such a procedure does not provide sufficient precision for its alignment using a SR beam. To solve this problem, we fabricated a special alignment head with four degrees of freedom (Fig. 4). The head is placed on a rotating goniometric module with a piezomotor previously developed by the authors [7]. This module provides lens alignment about the vertical axis. The system of step motors allows lens alignment along the horizontal axis and its displacement across the SR beam. The alignment head is incorporated into the general setup automation system and is remotely controlled.

The lens is positioned on the head so that its input plane would coincide with the vertical rotation axis of the alignment head. Certainly, the horizontal axis of the head intersects with its vertical axis. Such a lens position allows independent alignment over various degrees of freedom. Initially, the lens entrance aperture is brought to the collimated SR beam by transverse motions of the head. Then, a special program, rocking the lens along two axes, searches for lens "transmission," moving the lens exit aperture along a curve reminding a square spiral. It is important that the input lens plane is not displaced during such rocking, and the transverse alignment of the lens is not violated.

\section{MEASUREMENTS}

The developed device, i.e., the knife plus lens (described above) was used at the Kurchatov SR Center to measure the image profile of the radiation source

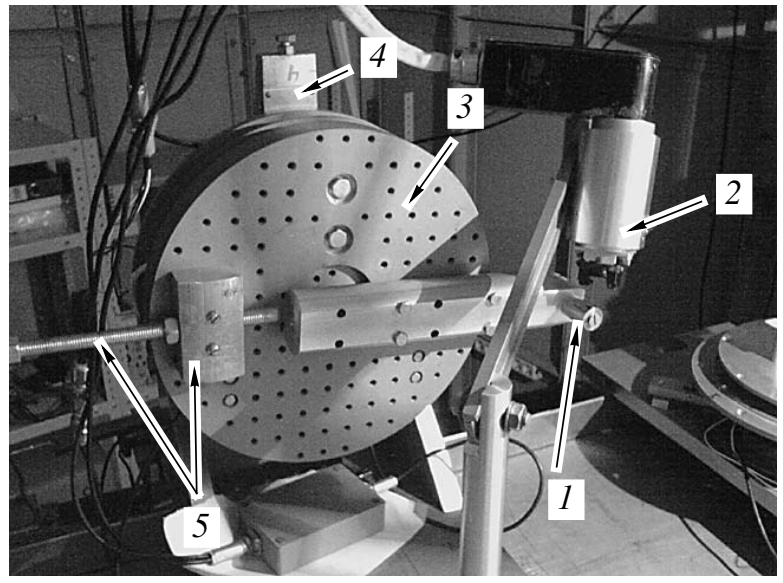

Fig. 2. X-ray knife: (1) the knife itself, i.e., cylindrical element, (2) detector of radiation scattered by the knife cylinder, (3) scanning module with piezoelectric column, (4) piezomotor of the rotating goniometric module, and (5) counterbalance.

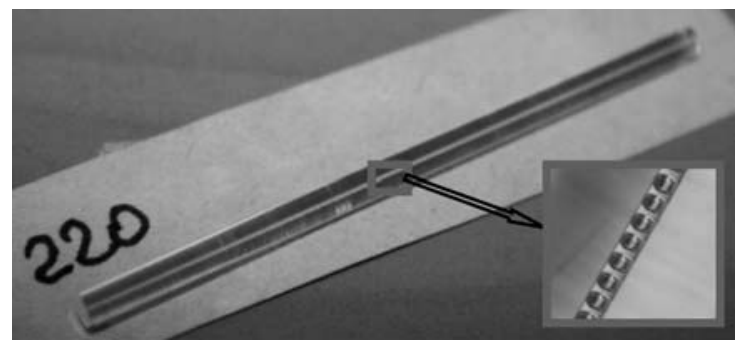

Fig. 3. X-ray refractive microcapillary lens. The surface curvature radius of an individual lens is $100 \mu \mathrm{m}$. The number of elementary lenses $N=220$.

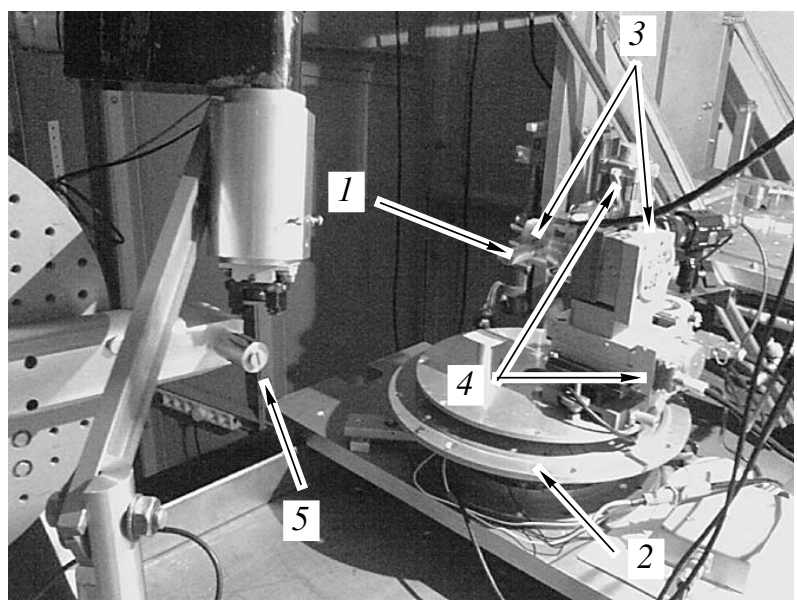

Fig. 4. Specialized goniometric head developed by the authors for adjusting the microcapillary lens: (1) microsupport for placing the microcapillary lens, $(2)$ rotating goniometric module (vertical axis), (3) rotating head (horizontal axis), (4) step motors of transverse motions, and (5) x-ray knife cylinder. 


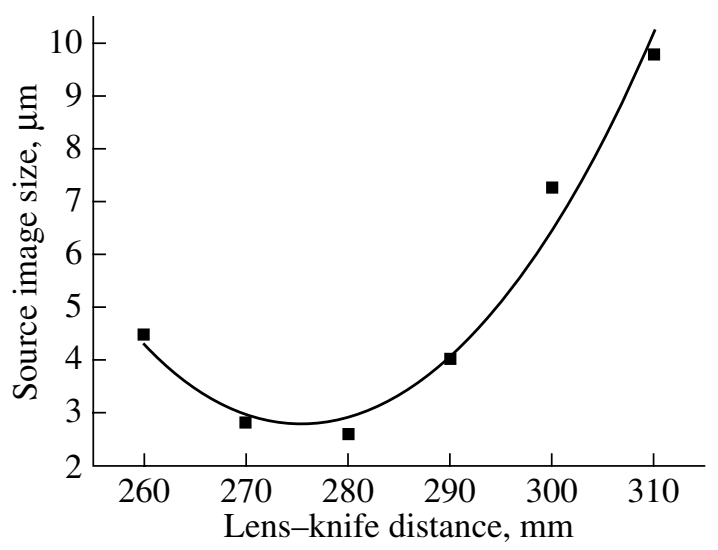

Fig. 5. Dependence of the measured size of the radiation source image on the distance between the lens and recorded image.

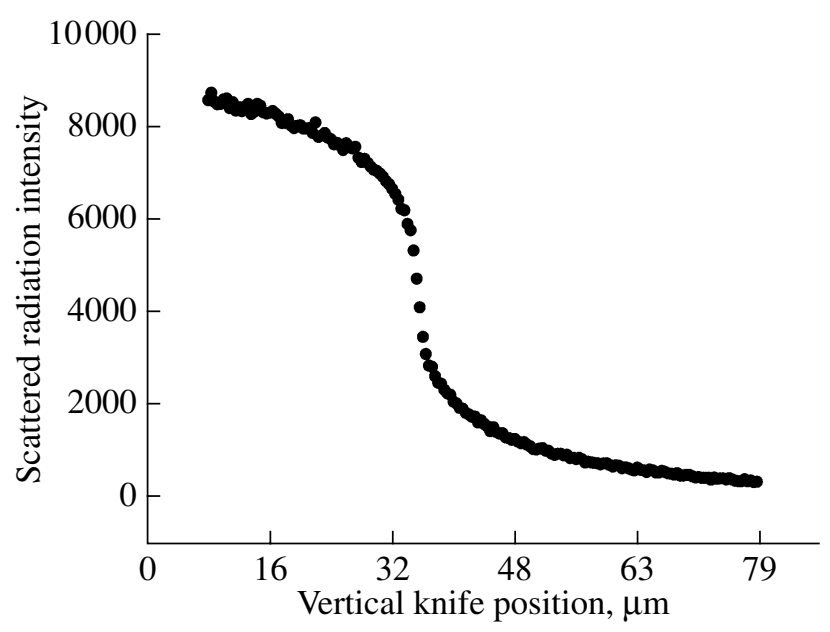

Fig. 6. Dependence of the intensity of radiation scattered by the $\mathrm{x}$-ray knife on the vertical knife position. The lens-knife distance is $280 \mathrm{~mm}$.

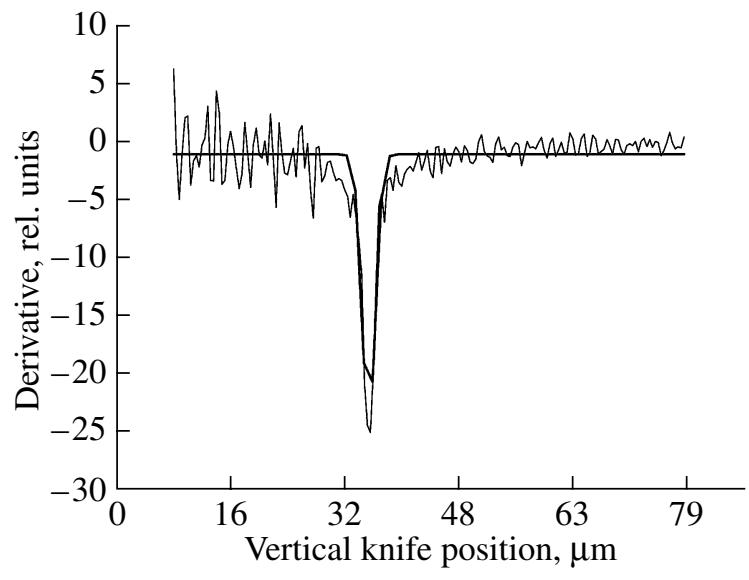

Fig. 7. Derivative of the intensity curve shown in Fig. 6 and corresponding fitting by the Gaussian. The full width at half maximum of the fitted curve $(2.4 \mu \mathrm{m})$ is the full width of the radiation source image in the $\mathrm{x}$-ray refractive lens. formed by an electron ring bunch with an energy of $2.5 \mathrm{GeV}$.

The $\mathrm{x}$-ray beam was formed at a storage ring current of $50 \mathrm{~mA}$ and an electron energy of $2.5 \mathrm{GeV}$. The distance from the emission point to the lens was $16200 \mathrm{~mm}$, the distance from the lens to the knife was varied during the experiment from 260 to $310 \mathrm{~mm}$. The photon energy was $18 \mathrm{keV}$. The dependence of the measured source image size on the distance between the lens and the measured image is shown in Fig. 5. Figure 6 shows the dependence of the intensity of radiation scattered by the $\mathrm{x}$-ray knife on its vertical position. The lens-knife distance is $280 \mathrm{~mm}$.

The width of the vertical cross section of the beam image profile, determined by differentiating the function of the scattered radiation intensity, was $2.4 \mu \mathrm{m}$ (Fig. 7). We note that such a small value of the focused x-ray spot was achieved at the Kurchatov SR source for the first time. Taking into account the ratio of the source-lens and lens-knife distances, the vertical size of the radiation source is $140 \mu \mathrm{m}$, which corresponds to the design parameters of the storage ring.

\section{CONCLUSIONS}

Current studies using SR are in many respects caused by a decrease in the studied material volume. The micrometer border was already crossed in the world practice; submicrometer samples are studied. The scanning microscopy resolution is directly controlled by the possibility of focusing the source radiation into a small spot. Namely the size of this spot controls the scanning microscope resolution.

The described device, i.e., the $\mathrm{x}$-ray knife, reduces the time of measurement for the vertical cross section of the spatial intensity distribution profile in the focused SR beam by an order of magnitude in comparison with recording using a high-resolution x-ray film. The use of automatics makes it possible to lower the role of subjectivity in estimation of the analyzed beam size. An important feature of the device is the use of a shading shutter of the round cylinder and two independent systems of its motion. Of principal importance is also the design and technological availability, low cost, and extremely simple service of the device under conditions of submicrometer resolution in the study of the spatial intensity distribution in x-ray beams.

\section{REFERENCES}

1. A. Snigirev, V. Kohn, I. Snigireva, and B. Lengeler, Nature 384, 49 (1996).

2. A. Snigirev, Rev. Sci. Instrum. 66, 2053 (1995).

3. O. Kurapova, S. Feste, M. Gather, et al., Proc. SPIE-Int. Soc. Opt. Eng. 5539, 38 (2004).

4. A. N. Artemiev, A. A. Snigirev, V. N. Korchuganov, et al., J. Surf. Investigation. X-Ray, Synchrotron and Neutron Techniques 1, 528 (2007). 
5. A. N. Artemiev, V. N. Korchuganov, A. G. Valentinov, et al., Nucl. Instrum. Methods Phys. Res. A 575, 269 (2007).

6. F. A. Hofmann, C. A. Freinberg-Trufas, S. W. Owens, et al., Nucl. Instrum. Methods Phys. Res. B 133, 145 (1997).

7. A. N. Artemiev, S. V. Krupin, V. D. Podshibyakin, et al., Nucl. Instrum. Methods Phys. Res. A 308, 459 (1991).

8. A. N. Artemiev, N. A. Artemiev, A. V. Zabelin, et al., Nucl. Instrum. Methods Phys. Res. A 575, 228 (2007).

9. Yu. I. Dudchik and N. N. Kolchevsky, Nucl. Instrum. Methods Phys. Res. A 421, 361 (1999).
10. Yu. I. Dudchik, N. N. Kolchevsky, F. F. Komarov, et al., Rev. Sci. Instrum. 75, 4651 (2004).

11. Yu. I. Dudchik, F. F. Komarov, M. A. Peistrup, et al., Spectrochim. Acta B 62, 598 (2007).

12. M. A. Piestrup, C. K. Gary, H. Park, et al., Appl. Phys. Lett. 86, 131104 (2005).

13. S. A. Pikuz, V. E. Asadchikov, K. M. Chandler, et al., Rev. Sci. Instrum. 74, 2247 (2003).

14. A. N. Artemev, N. A. Artemev, B. F. Kirillov, et al., Instr. Exp. Techn. 50, 539 (2007). 